\title{
Clinical relevance of blast-related traumatic brain injury
}

\author{
Geoffrey Appelboom • James Han • Sam Bruce • \\ Caroline Szpalski • E. Sander Connolly Jr.
}

Received: 19 July 2011 / Accepted: 12 October 2011 /Published online: 29 October 2011

(C) Springer-Verlag 2011

The global war against terrorism has created new challenges for neurosurgeons, craniofacial surgeons and maxillofacial surgeons; United States military operations in Iraq and Afghanistan have resulted in the greatest incidence of head trauma since the Vietnam conflict. The more frequent use of improvised explosive devices (IED), in conjunction with increased survival from improved body armor and battlefield medicine, has contributed to the increasing number of craniomaxillofacial injuries and consequent head trauma [28]. IEDs usually contain an explosive that, during detonation, is converted into a gas that rapidly expands and forms a high-pressure wave [4]. This blast overpressure wave travels at supersonic speeds and causes primary blast injury. The subsequent blast wind that follows the initial blast wave can propel objects, leading to further harm [7, 29].

The effect of a primary blast wave on the brain remains poorly understood despite the various models and computer simulations used to predict different mechanisms of injury $[5,27]$. Because $59 \%$ of the service members in Operation Iraqi Freedom (OIF) and Operation Enduring Freedom (OEF) suffered from TBI [21], a better understanding of

G. Appelboom $\cdot$ J. Han $\cdot$ S. Bruce $\cdot$ E. S. Connolly Jr. Department of Neurological Surgery, The Neurological Institute, Columbia University College of Physicians and Surgeons,

New York, NY 10032, USA

C. Szpalski

Department of Plastic and Craniofacial Surgery,

New York University (NYU),

New York, NY 10016, USA

G. Appelboom ( $\square)$

Department of Neurosurgery, Columbia University,

710 West 168th Street, Room 431,

New York, NY 10032, USA

e-mail: gappelbo@gmail.com mechanisms underlying primary blast injury and the establishment of guidelines of care are warranted. Persistent and debilitating symptoms are often associated with mild traumatic brain injury [24], underscoring the need for improved diagnostic and therapeutic modalities. Diffuse axonal injury (DAI) is widely hypothesized to be a principle mechanism of damage and the cause of persistent cognitive defects after traumatic brain injury (TBI). Angular forces can cause shearing or stretching of axons, leading to impaired axonal transport and swelling [9]. However, further elucidation of the pathological mechanism behind blast injury and determining the ideal diagnostic method to identify this condition may help lead to greater protection for military personnel and treatments for those already suffering from this disease.

The majority $(85 \%)$ of reported TBI cases are undetectable with imaging (computed tomography (CT) and magnetic resonance imaging (MRI) are usually insensitive to its characteristic small lesions $[1,13])$ and are classified as mild $[2,25]$ based on the Department of Defense (DoD) screening criteria. The DoD defines mild TBI as loss of consciousness, amnesia, mental status alteration at time of injury, and/or focal neurological deficit or peri-injury confusion/disorientation in a patient with a Glasgow Coma Scale (GCS) score of 13-15 [18]. Once diagnosed, mild TBI is a treatable disease typically associated with a very favorable prognosis [15]. As a result, the recently published results by Mac Donald and colleagues have created a sense of cautious optimism regarding the use of diffusion tensor imaging (DTI) in detecting structural brain damage in blast-exposed patients suffering from mild TBI.

In their study, Mac Donald et al. enrolled 63 patients, ages 19-58, from a screened population of 122 service members. The primary goal was to establish whether or not traumatic axonal injury is a primary feature of human blast- 
related TBI. As we previously mentioned, if such an investigation is more than necessary to improve patient care and outcomes, we have some major concerns about the validity of this study, particularly with regard to patient selection and inclusion criteria. First of all, the selected patient population had primary blast exposure plus another concurrent related injury to the head (fall, motor-vehicle crash, blow to the head by a blunt object) [16], which unfortunately is an important limiting factor, preventing the direct contribution of primary blast exposure to TBI from being precisely determined. Although they did not explicitly restrict patients based on the severity of their injury, patients with moderate to severe TBI were excluded by requiring an in-person informed consent. Another major limitation is the self-reported initial screening questionnaire used by the investigators. The majority of injured personnel possesses a strong desire to return to duty with their deployed unit in the combat zone, and may underreport their blast exposure and related symptoms. Despite the authors' intentions, they may have excluded patients with very mild degrees of TBI from their study. Mac Donald et al. also attempted to exclude patients' conditions that would alter DTI signals, but their enrolled patients did not undergo scanning prior to blast injury. The military personnel who uniquely make up the majority of victims suffering from blast injury are recruited and trained to work in high stress and physically demanding situations. These conditions can theoretically lead to repetitive brain injury prior to engagement on the battlefield, raising the possibility that their results stemmed from pre-existing conditions in addition to mild head trauma.

In addition to these limitations, we have further concerns regarding the study by Mac Donald et al. All patients underwent DTI scanning within 90 days after their injury. However, in another study, a follow-up DTI scan without clinical assessment in two patients with TBI revealed that some regions with initially detectable DAI were partially or completely corrected 30 days after injury [1]. Given the heterogeneity of TBI on an individual basis, a cellular repair mechanism may correct partial injury within a certain time period. As a result, a 90-day window may be too broad when attempting to initially identify patients with mild TBA. Moreover, only a third of patients with TBI had definitively abnormal scans when analyzed individually [16], demonstrating that DTI alone cannot be used to make a diagnosis with certainty, especially since numerous aspects about this technique are still poorly understood [10]. Therefore, diagnostic interpretation in the individual patient relying solely on DTI results remains problematic because substantial overlap in the range of DTI metrics exists between age-, gender- and education-matched controls and mild TBI patients $[11,22,26]$. Mac Donald et al. did not control for these factors in the study, though they do show there was no correlation with age in their cohort.
Another concern is the duration of loss of consciousness and post-traumatic amnesia, which, as Mac Donald and colleagues explicitly state, was not available or not reliable. Unfortunately, this may severely impair the assessment of their patients, and these are two very important criteria in the diagnosis of TBI that are used to divide this condition into categories of mild, moderate and severe. Furthermore, challenges arise when attempting to accurately identify mild TBI because of the overlap of post-concussion symptoms and post-traumatic stress disorder (PTSD). Military members serving in a combat zone are at risk for both of these, and they can affect the same patient [6]. This needs to be taken into consideration to ensure that all enrolled patients truly have mild TBI and not PTSD. Some even argue that the current screening procedures and definitions of mild TBI are flawed [12], further complicating matters.

Finally, the Mac Donald study used region of interest analysis to explicitly assess major orbitofrontal, posterior fossa white-matter tracts and other commonly affected regions using DTI. This method of analysis only samples small and specific stereotypical regions instead of examining the entire brain. Mild TBI lesions can occur throughout various regions of the brain, underestimating the impact and extent of TBI and limiting the clinical utility of this type of analysis [19]. Therefore, whole brain or voxel-based analysis may represent a better way to analyze DTI results since it is automated, removes operator bias and measures the overall extent of white matter injury [17]. A study by Niogi et al. considered white matter tracts damaged if the values were more than 2.5 SD below the mean of their control subjects [20]; the Mac Donald study, on the other hand, defined abnormalities as reductions of at least 2 SD below the mean. Based on their data, it seems that a large number of their patients would not have been categorized as having structural abnormalities if a more stringent cutoff point had been used.

Unfortunately there are only two other studies involving service members exposed to blast injury. Peskind and colleagues [23] compared 12 Iraq war veterans who were exposed to at least one blast that resulted in acute mild TBI and had persistent postconcussive symptoms with civilian controls. Veterans with blast exposure mild TBI showed consistent regional hypometabolism in certain regions of the brain, demonstrating that blast exposure resulting in mild TBI may have a neurobiological substrate that is measurable. In the only other published study using DTI, Levin and colleagues [14] examined the effects of mild to moderate blast-related TBI in $37 \mathrm{OIF} / \mathrm{OEF}$ veterans and service members, and compared them with a group of OIF/OEF veterans without blast exposure who sustained injury to other body regions or had no injury. This group found no between-group differences in white matter integrity. Unfortunately, there are limitations involved with both of these studies, and definitive conclusions cannot be reached solely based on their evidence. 
The majority of patients categorized with mild TBI have no abnormality on routine CT [3]. As a result, it is extremely difficult to define mild TBI on a radiological basis, and its clinical assessment is currently much more relevant. These obstacles highlight the continued need for future studies that explore blast-related injuries similar to the one conducted by Mac Donald and colleagues. Although their study has limitations, the results presented by the Mac Donald group show promise and sets a precedent upon which future studies can improve as we strive for a better understanding of blast exposure and TBI. An ideal study would integrate prior traumatic non-blastrelated lesions and acquire multiple DTI data sets prior to injury onset, prior to engagement in war areas and at short intervals beginning at onset. It would also be longitudinal in order to chart DAI progression in each patient over a certain timeline and be conducted in a prospective manner. Although existing evidence comes mostly from sports studies, neuropsychological tests have been demonstrated to be useful in the detection of cognitive impairment following mTBI $[3,8]$. As a result, neuropsychological evaluations should also be conducted in future studies to define a correlation with DTI results. This may help give stronger validation to the neuroradiological data and provide further information that may help make a better distinction between PTSD and mild TBI. These types of studies may eventually help us predict future changes in white matter that are characterized by DTI and correlate them with clinical outcome in patients. Hopefully, advances in neuroimaging modalities will improve our ability to detect clinically significant but sub-threshold structural abnormalities in blast-related mild TBI.

\section{Conflicts of interest None.}

\section{References}

1. Arfanakis K, Haughton VM, Carew JD, Rogers BP, Dempsey RJ, Meyerand ME (2002) Diffusion tensor MR imaging in diffuse axonal injury. AJNR Am J Neuroradiol 23:794-802

2. Bazarian JJ, McClung J, Shah MN, Cheng YT, Flesher W, Kraus J (2005) Mild traumatic brain injury in the United States, 1998200. Brain Inj 19:85-91

3. Borg J, Holm L, Cassidy JD, Peloso PM, Carroll LJ, von Holst H, Ericson K, WHO Collaborating Centre Task Force on Mild Traumatic Brain Injury (2004) Diagnostic procedures in mild traumatic brain injury: results of the WHO Collaborating Centre Task Force on Mild Traumatic Brain Injury. J Rehabil Med. (43 suppl):61-75

4. Brookings Institution, Saban Center for Middle East Policy (2010) Iraq index: tracking variables of reconstruction and security in post-Saddam Iraq http://www.brookings.edu/iraqindex. Accessed 20 Jun 2011

5. Chafi MS, Karami G, Ziejewski M (2010) Biomechanical assessment of brain dynamic responses due to blast pressure waves. Ann Biomed Eng 38:490-504
6. Dempsey KE, Dorlac WC, Martin K, Fang R, Fox C, Bennett B, Williams K, Flaherty S (2009) Landstuhl Regional Medical Center: traumatic brain injury screening program. J Trauma Nurs 16(1):6-7, 10-12

7. DePalma RG, Burris DG, Champion HR, Hodgson MJ (2005) Blast injuries. N Engl J Med 352:1335-1342

8. Echemendia RJ, Putukian M, Mackin RS, Julian L, Shoss N (2001) Neuropsychological test performance prior to and following sports-related mild related traumatic brain injury. Clin J Sport Med 11(1):23-31

9. Elder GA, Cristian A (2009) Blast-related mild traumatic brain injury: mechanisms of injury and impact on clinical care. Mt Sinai J Med 76(2):111-118

10. Gean AD, Fischbein NJ (2010) Head trauma. Neuroimaging Clin N Am 20(4):527-556

11. Hasan KM, Kamali A, Iftikhar A, Kramer LA, Papanicolaou AC, Fletcher JM, Ewing-Cobbs L (2009) Diffusion tensor tractography quantification of the human corpus callosum fiber pathways across the lifespan. Brain Res 1249:91-100

12. Hoge CW, Goldberg HM, Castro CA (2009) Care of war veterans with mild traumatic brain injury-flawed perspectives. N Engl J Med 360:1588-1591

13. Huisman TA, Schwamm LH, Schaefer PW, Koroshetz WJ, ShettyAlva N, Ozsunar Y, Wu O, Sorensen AG (2004) Diffusion tensor imaging as potential biomarker of white matter injury in diffuse axonal injury. AJNR Am J Neuroradiol 25:370-376

14. Levin HS, Wilde E, Troyanskaya M, Petersen NJ, Scheibel R, Newsome M, Radaideh M, Wu T, Yallampalli R, Chu Z, Li X (2010) Diffusion tensor imaging of mild to moderate blast-related traumatic brain injury and its sequelae. J Neurotrauma 27(4):683-694

15. Ling GS, Ecklund JM (2011) Traumatic brain injury in modern war. Curr Opin Anaesthesiol 24(2):124-130

16. Mac Donald CL, Johnson AM, Cooper D, Nelson EC, Werner NJ, Shimony JS, Snyder AZ, Raichle ME, Witherow JR, Fang R, Flaherty SF, Brody DL (2011) Detection of blast-related traumatic brain injury in U.S. military personnel. N Engl J Med 2;364 (22):2091-100

17. Marquez de la Plata CD, Yang FG, Wang JY, Krishnan K, Bakhadirov K, Paliotta C, Aslan S, Devous MD, Moore C, Harper C, McColl R, Munro Cullum C, Diaz-Arrastia R (2011) Diffusion tensor imaging biomarkers for traumatic axonal injury: analysis of three analytic methods. J Int Neuropsychol Soc 17(1):24-35

18. Mild Traumatic Brain Injury Committee of the Head Injury Interdisciplinary Special Interest Group of the American Congress of Rehabilitation Medicine (1993) Definition of mild traumatic brain injury. J Head Trauma Rehabil 8:86-87

19. Niogi SN, Mukherjee P (2010) Diffusion tensor imaging of mild traumatic brain injury. J Head Trauma Rehabil 25(4):241-255

20. Niogi SN, Mukherjee P, Ghajar J, Johnson C, Kolster RA, Sarkar R, Lee H, Meeker M, Zimmerman RD, Manley GT, McCandliss BD (2008) Extent of microstructural white matter injury in postconcussive syndrome correlates with impaired cognitive reaction time: a $3 \mathrm{~T}$ diffusion tensor imaging study of mild traumatic brain injury. AJNR Am J Neuroradiol 29:967-973

21. Okie S (2005) Traumatic brain injury in the war zone. New Engl J Med 352:2043-2047

22. Ota M, Obata T, Akine Y, Ito H, Ikehira H, Asada T, Suhara T (2006) Age-related regeneration of corpus callosum measured with diffusion tensor imaging. Neuroimage 31(4):1445-1452

23. Peskind ER, Petrie EC, Cross DJ, Pagulayan K, McCraw K, Hoff D, Hart K, Yu CE, Raskind MA, Cook DG, Minoshima S (2011) Cerebrocerebellar hypometabolism associated with repetitive blast exposure mild traumatic brain injury in 12 Iraq war veterans with persistent post-concussive symptoms. Neuroimage 54(Suppl 1): S76-S82

24. Plurad DS (2011) Blast injury. Mil Med 176(3):276-282 
25. Saatman KE, Duhaime AC, Bullock R, Maas AI, Valadka A, Manley GT, Workshop Scientific Team and Advisory Panel Members (2008) Classification of traumatic brain injury for targeted therapies. J Neurotrauma 25:719-738

26. Sullivan EV, Pfefferbaum A (2006) Diffusion tensor imaging and aging. Neurosci Biobehav Rev 30(6):749-761

27. Taylor PA, Ford CC (2009) Simulation of blast-induced early-time intracranial wave physics leading to traumatic brain injury. J Biomech Eng 131:061007

28. Wade AL, Dye JL, Mohrle CR, Galarneau MR (2007) Head, face, and neck injuries during Operation Iraq Freedom II: results from the US Navy-Marine Corps Combat Trauma Registry. J Trauma 63:836-840

29. Wolf SJ, Bebarta VS, Bonnett CJ, Pons PT, Cantrill SV (2009) Blast injuries. Lancet 374:405-415

\section{Comment}

Appelboom and colleagues direct their attention to the recently published paper of Mac Donald et al. about the detection of blastrelated traumatic brain injury in U.S. military personnel [1]. Recent publications showed, in fact, that $60 \%$ of the service members in war zones suffered from any type of TBI [2]. The majority of them, like other types of TBI, presented generally lesions not detectable with CT-scan, with white matter damage and diffuses axonal injury. As consequence, neuropsychological impairments will occur with documented relationships between cognitive impairments and DTI damage white matter injury, mainly in the parahippocampal gyrus, left inferior parietal lobe, and left anterior cingulate [3].

Overall we agree with the main concerns expressed by Appelboom and colleagues, which reflect the issues of part of the neurosurgical community. In particular, Appelboom and colleagues are concerned about the 90-day window to perform follow-up DTI scan, which seems to be too broad. Moreover, we are uncertain if the selected assessed regions of interest analyzed are significant in such type of study.

Additionally, although the study of Mac Donald and colleagues may improves the data of mild traumatic brain injury by blasts of improvised explosive devices, we think that this study has failed a chance. In detail, the study was not conducted in a prospective matter. Unlike car accidents or similar other cause of TBI, the military personnel is recruited and trained to work in military areas. Such type of military personnel undergoes strong physical and mental preparation, which can theoretically have as consequence repetitive brain injury, due to the very strong actions in the training time. To exclude such types of injuries, the personnel should undergo to DTI scan before the engagement in war areas. Hence the controls were more trustworthy.

Another point of concern in the study of Mac Donald and colleagues is the absence of extensive neuropsychological evaluations correlated with the DTI informations. This art of correlation may corroborate the neuroradiological data and give more interpretations especially to understand the PTSD consequences.

In conclusion, we agree with the concerns expressed by Appelboom and colleagues over the cited study, in particular over the optimism about the expected predictive value of DTI in detecting structural brain damage. On the other side, the study of Mac Donald and colleagues may offer a chance to establish more extensive and structured studies in the- until now- poorly understood field of mild TBI with axonal diffuse injury, with useful data valuable not only in blast-related brain injury but also in civilian TBI like traffic accidents, hockey, football, and more.

Alex Alfieri

David Hoza

Jens Rachinger

Halle (Saale), Germany

1. Mac Donald CL, Johnson AM, Cooper D, Nelson EC, Werner NJ, Shimony JS, Snyder AZ, Raichler ME, Witherow JR, Fang R, Flaherty SF, Brody DL (2011) Detection of blast-related traumatic brain injury in U.S. military personnel. N Engl J Med 364(22):2091-100

2. Okie S (2005). Traumatic brain injury in the war zone. N Engl J Med ;352(20):2043-7.

3. Kondo K, Maruishi M, Ueno H, Sawada K, Hashimoto Y, Ohshita T, Takahashi T, Ohtsuki T, Matsumoto M (2010) The pathophysiology of prospective memory failure after diffuse axonal injury-lesion-symptom analysis using diffusion tensor imaging. BMC Neurosci 11:147. 